\title{
The effect of essential oils on methicillin-resistant Staphylococcus aureus using a dressing model
}

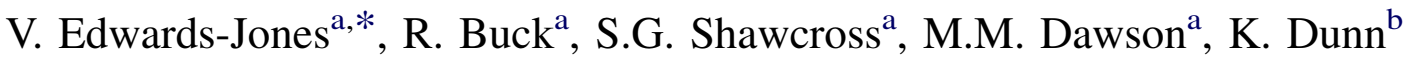 \\ ${ }^{\mathrm{a}}$ Department of Biological Sciences, the Manchester Metropolitan University, Chester Street, Manchester, M15GD, UK. \\ ${ }^{\mathrm{b}}$ Burns Centre, Acute Block, Wythenshawe Hospital, Southmoor Road, Manchester, M239LT, UK.
}

Accepted 4 June 2004

\begin{abstract}
Patchouli, tea tree, geranium, lavender essential oils and Citricidal ${ }^{\mathrm{TM}}$ (grapefruit seed extract) were used singly and in combination to assess their anti-bacterial activity against three strains of Staphylococcus aureus: Oxford S. aureus NCTC 6571 (Oxford strain), Epidemic methicillin-resistant S. aureus (EMRSA 15) and MRSA (untypable). The individual essential oils, extracts and combinations were impregnated into filter paper discs and placed on the surface of agar plates, pre-seeded with the appropriate strain of Staphylococcus. The effects of the vapours of the oils and oil combinations were also assessed using impregnated filter paper discs that were placed on the underside of the Petri dish lid at a distance of $8 \mathrm{~mm}$ from the bacteria. The most inhibitory combinations of oils for each strain were used in a dressing model constructed using a four layers of dressings: the primary layer consisted of either Jelonet ${ }^{\mathrm{TM}}$ or TelfaClear $^{\mathrm{TM}}$ with or without Flamazine $^{\mathrm{TM}}$; the second was a layer of gauze, the third a layer of Gamgee and the final layer was Crepe bandage. The oil combinations were placed in either the gauze or the Gamgee layer. This four-layered dressing was placed over the seeded agar plate, incubated for $24 \mathrm{~h}$ at $37{ }^{\circ} \mathrm{C}$ and the zones of inhibition measured. All experiments were repeated on three separate occasions. No anti-bacterial effects were observed when Flamazine ${ }^{\mathrm{TM}}$ was smeared on the gauze in the dressing model. When Telfaclear ${ }^{\mathrm{TM}}$ was used as the primary layer in the dressing model compared to Jelonet $^{\mathrm{TM}}$, greater zones of inhibition were observed. A combination of Citricidal ${ }^{\mathrm{TM}}$ and geranium oil showed the greatest-antibacterial effects against MRSA, whilst a combination of geranium and tea tree oil was most active against the methicillin-sensitive $S$. aureus (Oxford strain). This study demonstrates the potential of essential oils and essential oil vapours as antibacterial agents and for use in the treatment of MRSA infection.
\end{abstract}

(C) 2004 Elsevier Ltd and ISBI. All rights reserved.

Keywords: MRSA; Wound infection; Dressings; Essential oils

\section{Introduction}

Despite major advances in wound management, infection still remains an important factor in wound healing. In burns, approximately $75 \%$ of deaths are due to complications with sepsis resulting from wound infection [1]. Among other adverse effects, infection delays healing, contributes to graft failure and can increase the depth of a burn. Approximately $30 \%$ of burn wounds become colonised with Staphylococcus aureus [2] and outbreaks of methicillin-resistant $S$. aureus (MRSA) have created major problems for burn units and

\footnotetext{
* Corresponding author. Tel.: +0161 247 1229; fax: +0161 2476325 .

E-mail address: v.e.jones@mmu.ac.uk (V. Edwards-Jones).
}

intensive care units in terms of cross-infection and rehabilitation of the patient due to imposed barrier nursing [3]. Some MRSA strains, termed epidemic MRSA (EMRSA), have the ability to spread rapidly among patients and the dominant clonal EMRSA types 15 and 16 are problematic in the UK $[4,5]$. A new strain, EMRSA 17 , has recently been described in the UK [6]. Whilst vancomycin is one of the few remaining effective systemic antibiotics available for treatment, resistance has been reported and there is major concern that total antibiotic-resistant strains may emerge in the immediate future [7]. At present, topical anti-microbial therapy is the single most important component of wound care to prevent infection [8] and in hospitalised burn patients, Flamazine ${ }^{\mathrm{TM}}$ is by far the most frequently used 
topical prophylactic agent [9] but does not always penetrate into the wound [10] and cannot be used to eradicate carriage from the patient or the environment.

Alternative therapies are being sought for treatment of MRSA and one area of interest is the use of essential oils. MRSA is susceptible to tea tree oil [11] but there are concerns about its toxicity [12]. Tea tree oil has been accepted as a powerful antibacterial agent [13] and is sold commercially in a wide range of products. There are hundreds of other essential oils available for use, many with known antibacterial properties. These oils contain numerous constituents that contribute to the characteristic odour and medicinal effects. The major chemical components that account for the pleasant aromatic odours are primarily terpenenes, monoterpenes and linalool [14]. The presence and quantity of the various components varies between oils and determines the individuality of the oil [15]. Although essential oils are known for their antimicrobial properties, medical teams rarely use them. This is primarily due to lack of scientific evidence of their efficacy, toxicity issues and the availability of conventional therapy. In this study, a range of essential oils and extracts were tested for their anti-bacterial properties using an in vitro dressing model to assess the antimicrobial action of the vapours.

\section{Materials and methods}

\subsection{Bacterial strains}

Three strains of $S$. aureus were used: an antibioticsusceptible strain, S. aureus NCTC 6571 (Oxford strain); a methicillin-resistant $S$. aureus (non-typable) and a methicillin-resistant $S$. aureus (phage type15). The two strains of MRSA were isolated from the wounds of two burn patients on the Burns Unit, Withington Hospital, Manchester, UK.

\subsection{Essential oils}

Four essential oils were used in the study: tea tree (Melaleuca alternifolia), patchouli (Pogostemon cablin), lavender (Lavendula officinalis), geranium (Pelargonium graveolens) (Supplier: Essentially Oils, Chipping Norton, UK); Citricidal $^{\mathrm{TM}}$ (grapefruit seed extract), a commercially available antibacterial agent, was also used.

\subsection{Assessment of antibacterial action using impregnated filter paper discs}

Aliquots $(20 \mu \mathrm{l})$ of each of the four oils or extract and their combinations (50:50) were spotted onto $5 \mathrm{~mm}$ filter paper discs and allowed to air dry for $40 \mathrm{~min}$. The discs were then placed onto the surface of sensitivity test agar (STA) (Lab M, Bury, UK) that had been seeded with a suspension of $10^{5}$ organisms $/ \mathrm{ml}$ of each strain of Staphylococcus (direct contact). Another set of seeded plates was exposed to the vapours of the oils or combinations by placing an impregnated disc in the centre of the lid of the Petri dish (vapour contact). All plates were incubated at $37{ }^{\circ} \mathrm{C}$ for $24 \mathrm{~h}$ and zones of inhibition (diameter in $\mathrm{mm}$ ) measured on the agar surface. All experiments were carried out three times.

\subsection{In vitro dressing model}

Aliquots $(100 \mu \mathrm{l})$ of essential oil and combinations that showed antimicrobial activity in the disk diffusion experiments were placed onto a small central area of either the Gamgee or gauze dressing layers. STA plates were seeded with a suspension of $10^{5}$ organisms $/ \mathrm{ml}$ of each strain of $S$. aureus and covered with four layers of dressings as shown in Fig. 1. Modifications were made to the primary layer by constructing a model with or without Flamazine ${ }^{\mathrm{TM}}$ and using either Telfa Clear ${ }^{\mathrm{TM}}$ or Jelonet ${ }^{\mathrm{TM}}$, or both, which are reduced adherence dressings. The in vitro models were then incubated at $37^{\circ} \mathrm{C}$ for $24 \mathrm{~h}$ and the zones of inhibition measured. All experiments were carried out three times.

\section{Results}

\subsection{Antibacterial action of single oils: direct contact}

All bacterial strains showed some susceptibility to each individual essential oil when tested in direct contact using

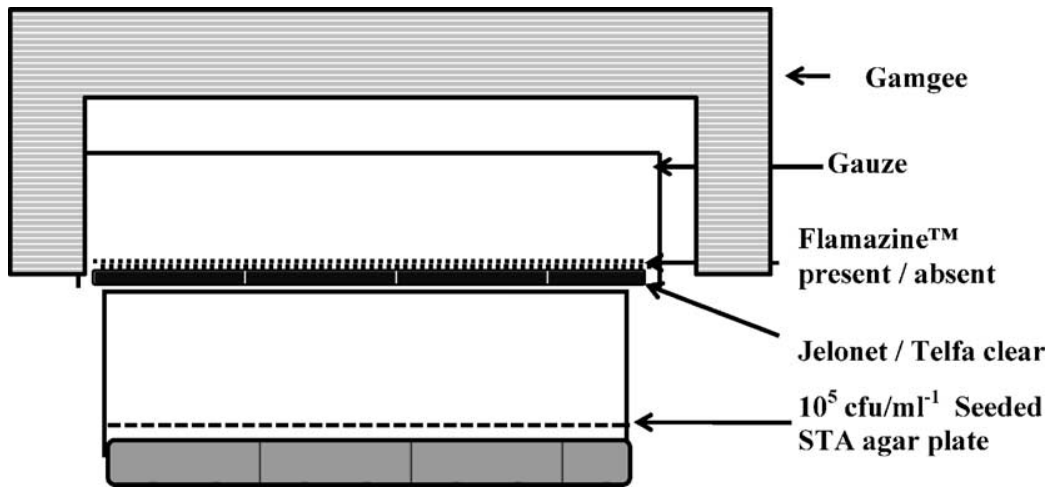

Fig. 1. The construction of the in vitro dressing model. 


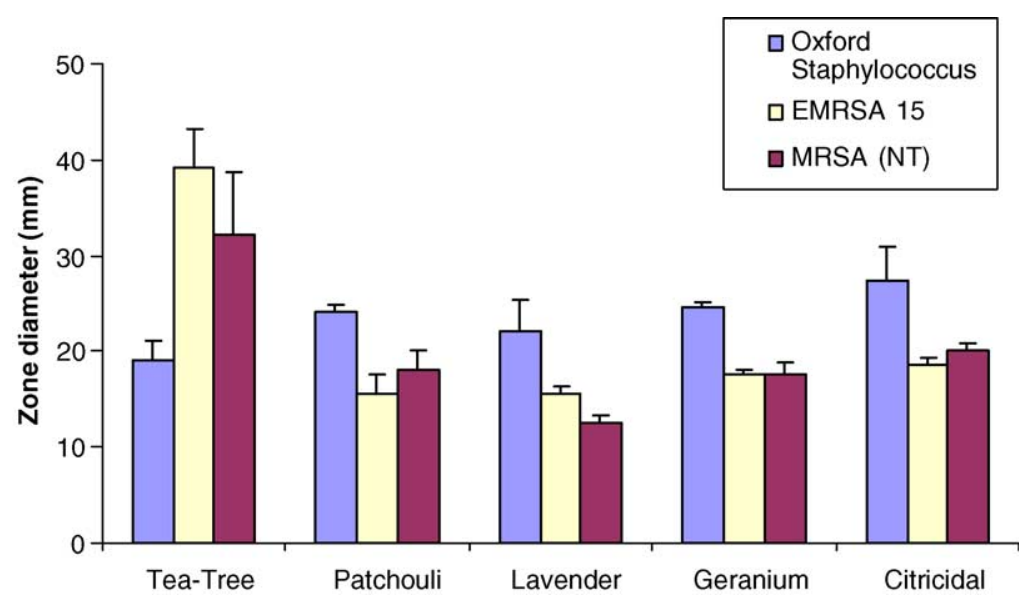

Fig. 2. Zones of inhibition of three strains of Staphylococcus aureus tested against individual essential oils placed in direct contact.

the disc diffusion method. The size of the zone of inhibition varied depending upon the oils and the strain of $S$. aureus used. Tea tree oil gave the largest zones of inhibition when tested against MRSA than any of the other oils. MRSA appeared to be more susceptible to tea tree oil than the Oxford Staphylococcus. Patchouli, lavender, geranium and Citricidal $^{\text {TM }}$ inhibited growth of the three strains of staphylococcus, but the Oxford Staphylococcus showed larger zones of inhibition (Fig. 2).

\subsection{Antibacterial action of single oils: vapour phase}

The Oxford Staphylococcus was most susceptible to the vapours of tea tree, patchouli and geranium oil, but not to lavender and Citricidal ${ }^{\mathrm{TM}}$. The MRSA strains showed no susceptibility to the vapours apart from in one strain, where tea tree oil showed limited effect (Fig. 3).

\subsection{Antibacterial action of oil combinations: direct contact}

Pair-wise combinations of these oils were tested for their antibacterial action. Where the disks were placed in direct contact with strains of staphylococcus, five of these combinations had little or no effect on the antibacterial activity

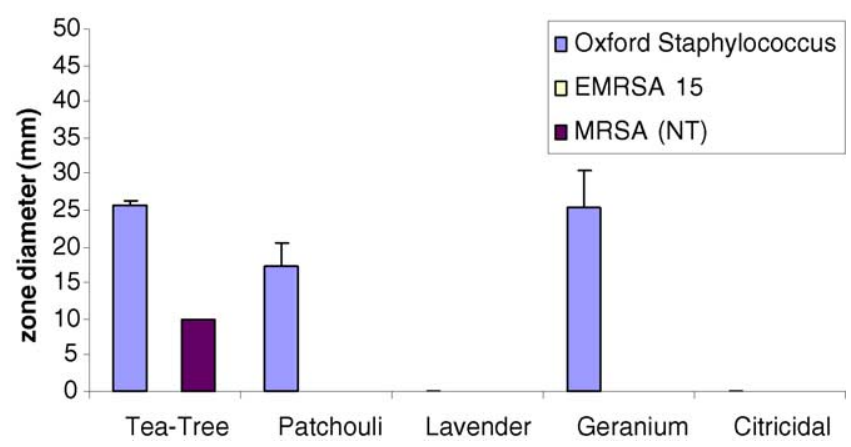

Fig. 3. Zones of inhibition of three strains of Staphylococcus aureus tested against individual essential oils in vapour contact. (patchouli and lavender, patchouli and Citricidal ${ }^{\mathrm{TM}}$, patchouli and geranium, lavender and Citricidal ${ }^{\mathrm{TM}}$, and geranium and Citricidal $^{\mathrm{TM}}$ ) compared to the single oil. However, the other five combinations (patchouli and tea tree, lavender and geranium, lavender and tea tree, grapefruit and tea tree and geranium and tea tree) produced increased zones of inhibition with the Oxford Staphylococcus, but not in the MRSA strains. In the MRSA strains, two combinations (lavender and tea tree, Citricidal $^{\mathrm{TM}}$ and tea tree) showed decreased activity when compared to tea tree oil alone (Fig. 4).

\subsection{Antibacterial action of oil combinations: vapour phase}

In the vapour phase, there were marked differences in observed antimicrobial effect of certain oil combinations; this was dependent upon the oil combination used and the strain of staphylococcus. Increased zones of inhibition were observed with the two MRSA strains when combinations of patchouli and tea tree, Citricidal ${ }^{\mathrm{TM}}$ and geranium, and Citricidal $^{\mathrm{TM}}$ and tea tree were used (Fig. 5). Whereas with single oils, no zones of inhibition or very small zones were noted (Fig. 3)

\subsection{In vitro dressing model}

Only the most effective oil combinations for each strain were used to test the dressing model. No inhibition of any of the strains by the oils occurred in the presence of Flamazine $^{\mathrm{TM}}$. In the absence of Flamazine ${ }^{\mathrm{TM}}$, there were noticeable zones of inhibition for the oil combinations. The MRSA strains were most susceptible to a combination of Citricidal $^{\mathrm{TM}}$ and geranium oil. The Oxford Staphylococcus was susceptible to a combination of geranium and tea tree oil. The zones of inhibition were increased when Telfa Clear $^{\mathrm{TM}}$ was used as the primary layer in place of Jelonet ${ }^{\mathrm{TM}}$ (Table 1). Fig. 6 demonstrates the effect of combinations of essential oil vapours (Citricidal ${ }^{\mathrm{TM}}$ and geranium) on an inoculum of $10^{5} \mathrm{cfu} / \mathrm{ml}$ of MRSA. 


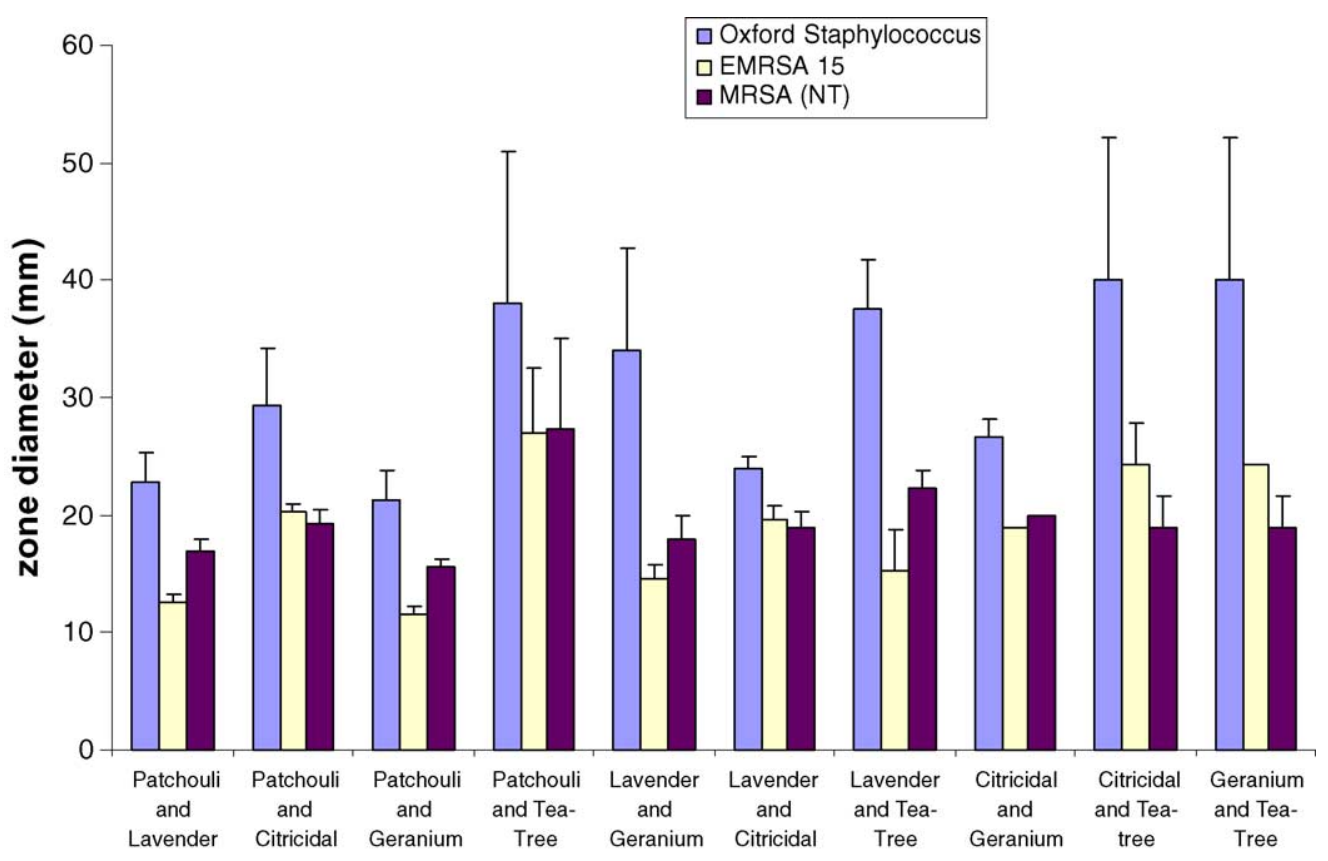

Fig. 4. Zones of inhibition of three strains of Staphylococcus aureus tested against combinations of essential oils placed in direct contact.

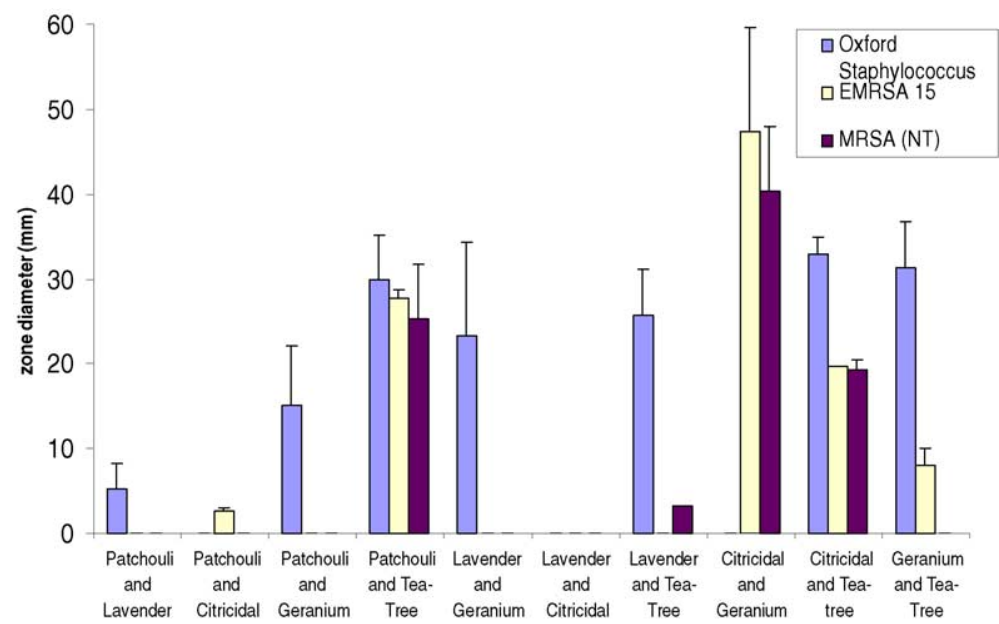

Fig. 5. Zones of inhibition of three strains of Staphylococcus aureus tested against combinations of essential oils placed in vapour contact.

\section{Discussion}

This study highlights the potential use of essential oil impregnated dressings for treating wounds infected with MRSA. Although the results are very promising, one must advise caution with their use on burned or open wounds. The toxicity and effect on re-epithelialisation of these compounds have not been investigated in this study: these are important issues for future research.

Several methods were tested to screen the oils for their antimicrobial effect. In previous studies, we have used minimal inhibitory concentrations (MICs) to report the antimicrobial action (data not shown): this is a common method used by other workers [16,17]. Although this technique is very accurate, it does not allow the effect of vapour phase to be tested. Using disc diffusion, the oil could be tested in direct contact with the bacteria and also in the vapour phase. It was also possible to test both properties using combinations of oils. In this study, we used a constant volume of oil $(20 \mu \mathrm{l})$ soaked into filter paper discs and a standard number of microorganisms seeded onto agar plates. This was set up to compare the effect of the oils on two strains of MRSA and a standard antibiotic-susceptible strain (Oxford Staphylococcus). When the oils were placed in direct contact with the bacteria, some inhibition of growth was seen irrespective of the oil, oil combination or the strain of Staphylococcus used. Tea tree oil is well known for its antiseptic properties and in this study it proved to be the most effective at inhibition of MRSA, when used as a single oil. When tea tree oil was used in combination with other oils, 
Table 1

Zones of inhibition $(\mathrm{mm})$ of three strains of Staphylococcus aureus tested against essential oils placed on either Gamgee or gauze, in the absence of Flamazine $\mathrm{TM}^{\mathrm{TM}}$ within a dressing model

\begin{tabular}{|c|c|c|c|c|c|}
\hline & \multirow[t]{3}{*}{ Essential oil } & \multicolumn{2}{|l|}{ TelfaClear $^{\mathrm{TM}}$} & \multicolumn{2}{|l|}{ Jelonet $^{\mathrm{TM}}$} \\
\hline & & Gamgee & Gauze & Gamgee & Gauze \\
\hline & & Mean (S.D.) & Mean (S.D.) & Mean (S.D.) & Mean (S.D.) \\
\hline Oxford staphylococcus & Tea tree and geranium & $58(37.2)$ & $81(1.0)$ & $39.3(12.8)$ & $32.6(10.6)$ \\
\hline MRSA (untypable) & Citricidal $^{\mathrm{TM}}$ and geranium & $64.6(8.4)$ & $72.6(2.5)$ & $44(8.5)$ & $36.8(11.7)$ \\
\hline EMRSA $15^{\circ}$ & Citricidal $^{\mathrm{TM}}$ and geranium & $70.6(5.1)$ & $77.6(0.6)$ & $41(6.6)$ & $34.6(16.1)$ \\
\hline Oxford staphylococcus & Patchouli and Tea tree & $\mathrm{NI}$ & $18(1.41)$ & NI & $\mathrm{NI}$ \\
\hline MRSA (untypable) & Patchouli and Tea tree & $\mathrm{NI}$ & $61.5(2.12)$ & NI & $58.5(2.12)$ \\
\hline EMRSA 15 & Patchouli and Tea tree & NI & $17.5(3.5)$ & NI & $22.5(3.53)$ \\
\hline Oxford staphylococcus & Citricidal $^{\mathrm{TM}}$ and Tea tree & NI & $14.5(0.70)$ & NI & Fine growth but no defined zone \\
\hline MRSA (untypable) & Citricidal $^{\mathrm{TM}}$ and Tea tree & NI & Fine growth but no defined zone & NI & Fine growth but no defined zone \\
\hline EMRSA 15 & Citricidal $^{\mathrm{TM}}$ and Tea tree & NI & Fine growth but no defined zone & NI & Fine growth but no defined zone \\
\hline
\end{tabular}

$\mathrm{NI}=$ No inhibition.

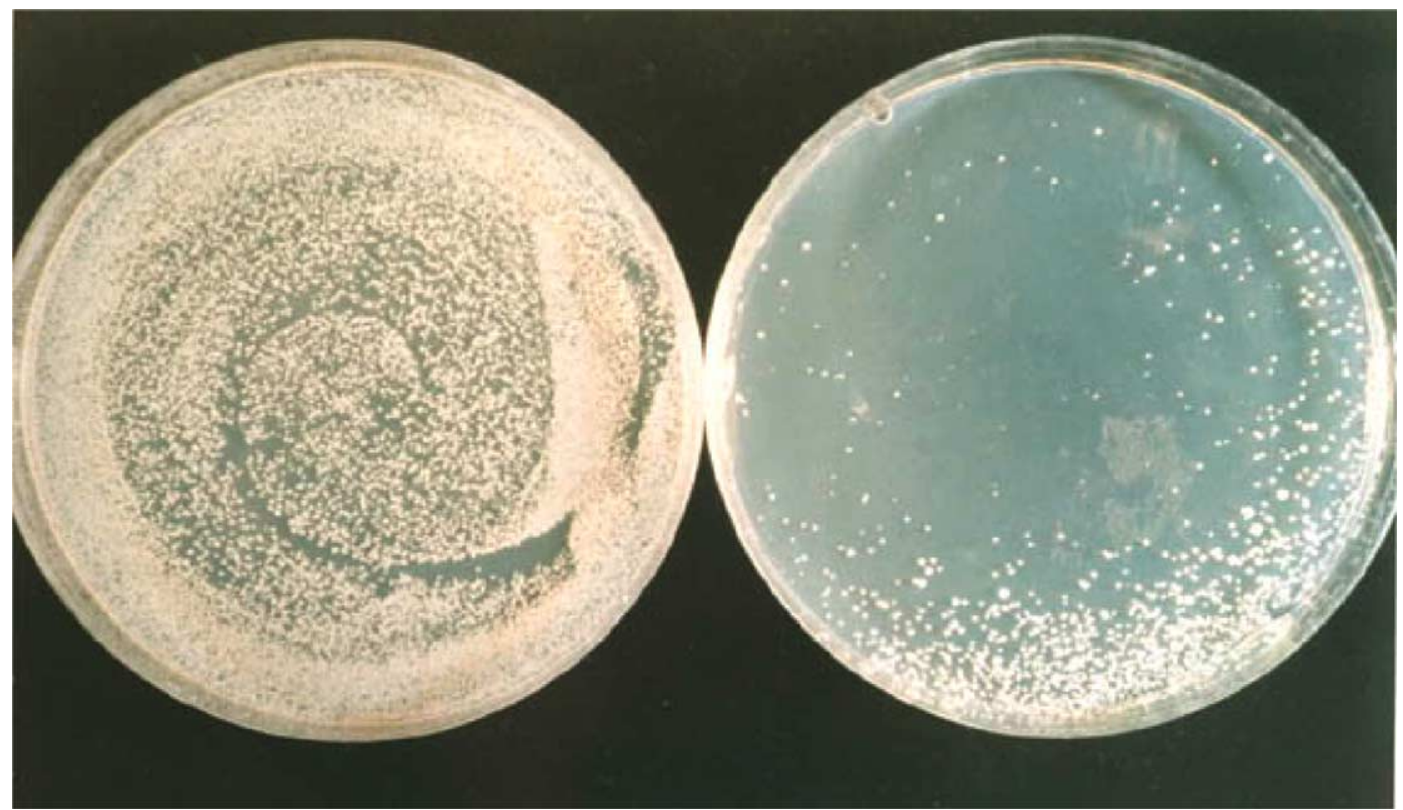

Fig. 6. This demonstrates the effect of combinations of essential oil vapours on an inoculum of $10^{5} \mathrm{cfu} / \mathrm{ml}$ of MRSA. The culture on the right is killed after $24 \mathrm{~h}$ exposure whilst the culture on the left demonstrates the density of the inoculum.

the susceptibility of Oxford Staphylococcus increased, but the susceptibility of all other strains decreased. Whether this is a result of action on different target sites within the strains has not been investigated in this study. It is possible that some of the single component presents in the complex mixture of the oils may have interacted to cause the observed effects. Extensive chemical analysis will have to be done to try to understand the precise nature of these effects. Studies on the mode of action of essential oils are not widely reported but work on tea tree oil has shown the bacterial cell membrane is disrupted and leakage of potassium has been observed [17]. There may be subtle differences in the membrane and/or the envelope of the different strains tested that accounts for the observed phenomena. Chemical analysis of the oils and oil combinations may determine whether there has been an additive effect of individual components within the oils or whether chemical changes have occurred to these components.

When exposed to the vapours of the oils and oil combinations, the inhibitory effects were markedly different from those found with direct contact. Only a small number of the oils and oil combinations vapours showed any activity against the staphylococcal strains. The zones of inhibition were more difficult to measure and invariably asymmetrical, resulting in large standard deviations. Of the single oils used, only tea tree oil vapour produced any zones of inhibition and this was only noticeable in the Oxford Staphylococcus. One oil combination (patchouli and tea tree) produced zones of inhibition with all the strains tested. This was interesting as neither of these oils alone showed any effect on the MRSA strains in the vapour phase. Geranium and Citricidal ${ }^{\mathrm{TM}}$ was the most effective combination of oils against the MRSA 
strains in the vapour phase, but this was not as effective against the Oxford Staphylococcus. From these observed effects, it would appear that certain chemical components of the oils resulting from the two single oils being mixed together may be increased work synergistically or cause release into the vapour phase and have some antibacterial action. More understanding of the complex chemistry of essential oils is required to unravel these phenomena.

The effects of the vapours of the most effective combinations were investigated in a dressing model that was constructed over a Petri dish that had been seeded with the different strains of staphylococcus. We demonstrated that the dressings and the topical antimicrobial preparations interfered with the observed effect of the oils. No inhibitory effects of the oils were observed in the presence of Flamazine $^{\mathrm{TM}}$. This was probably due to the thick coating of cream preventing the volatile compounds from affecting the $S$. aureus. Generally, when Telfa Clear ${ }^{\mathrm{TM}}$ was used instead of Jelonet ${ }^{\mathrm{TM}}$, there were larger zones of inhibition in all strains tested. Jelonet ${ }^{\mathrm{TM}}$, which contains impregnated paraffin, may have affected the vapour penetration.

In the dressing model the oils were placed in the centre portion of either the Gamgee or the gauze. There was little difference in the size of the zones of inhibition, whichever layer contained the oils: this is a promising finding. If future research shows that essential oils have a use in the clinical situation, it should be possible to leave the inner dressing in place and change only the outermost dressing and /or reimpregnate with oil(s). This means that the wound would not be unduly disturbed whilst the normal wound healing processes occur, but antimicrobial therapy can be maintained.

Several conclusions can be drawn from this study. We have shown that essential oils can have different effects on different strains of the same bacterial species. Some combinations of essential oils produce a greater antibacterial effect than the single oils alone when in contact with the bacteria. The vapour from essential oil combinations can also have a greater antibacterial effect than the individual oils and can be different from that seen when in direct contact with the bacteria. The vapour from some combinations of essential oils can inhibit MRSA, which may prove useful in eradicating them from a wound. The dressing model showed that the vapours of essential oils were prevented from inhibiting the growth of $S$. aureus strains in the presence of Flamazine ${ }^{\mathrm{TM}}$. The inhibitory effect was reduced in the presence of Jelonet ${ }^{\mathrm{TM}}$, compared to Telfa Clear $^{\mathrm{TM}}$.

\section{References}

[1] Lawrence JC. Burn bacteriology during the last 50 years. Burns 1992;18(suppl 2):23-9.

[2] Childs C, Edwards-Jones V, Heathcote DM, Dawson M, Davenport P. Patterns of Staphylococcus aureus colonisation, toxin production, immunity and illness in burned children. Burns 1994;20:514-21.

[3] Voss A, Milatovic D, Wallrauch-Schwarz C, Rosdahl VT, Braveny I. Methicillin-Resistant Staphylococcus aureus in Europe. Eur J Clin Microbiol Infect Dis 1994;13:50-5.

[4] Walker J, Borrow R, Edwards-Jones V, Oppenheim BA, Fox AJ. Epidemiological characterisation of methicillin-resistant Staphylococcus aureus isolated in the North West of England by protein A (spa) and coagulase (coa) gene polymorphisms. Epidemiol Infect 1998;121: 507-514.

[5] Anon. Methicillin-resistant Staphylococcus aureus. Commun Dis Rep Weekly 1998;8:372.

[6] Aucken HM, Ganner M, Murchan S, Cookson BD, Johnson AP. A new UK strain of epidemic methicillin-resistant Staphylococcus aureus (EMRSA-17) resistant to multiple antibiotics. J Antimicrob Chemother 2002;50:171-5.

[7] Smith TL, Pearson MC, Wilcox R, Cruz C, Lancaster MV, RobinsonDunn B E . Emergence of vancomycin resistance in Staphylococcus aureus. Glycopeptide-Intermediate Staphylococcus aureus working group. New Engl J Med 1999;340:493-501.

[8] Lawrence JC. Dressings and wound infection. Am J Surg 1994;167(suppl 1A):21S-4S.

[9] Edwards-Jones V, Dawson MM, Childs C. A survey into TSS in UK Burns Units. Burns 2000;26:323-33.

[10] Herruzo-Cabrera R, Vizcaino-Alcaide MJ, Mayer RF, Rey-Calero J. A new in vitro model to test the effectiveness of topical antimicrobial agents. Use of an artificial eschar. Burns 1992;18:35-8.

[11] Carson CF, Cookson BD, Farrelly HD, Riley TV. Susceptibility of methicillin-resistant S.aureus to the essential oil of Melaleuca alternifolia. J Antimicrob Chemother 1995;35:421-4.

[12] Faoagali J, George N, Leditschke JF. Does tea tree oil have a place in the topical treatment of burns? Burns 1997;23(4):349-51.

[13] Pattnaik S, Subramanyam VR, Kole C. Antimicrobial and antifungal activity of ten essential oils in vitro. Microbios 1996;86:237-46.

[14] Williams DG. The Chemistry of Essential Oils. Micelle Press; 1997.

[15] Tisserand R, Balacs T. Essential Oil Safety: A Guide for Health Care Professionals.. London: Churchill Livingstone; 1996.

[16] Carson CF, Hammer KA, Riley TV. Broth micro-dilution method for determining the susceptibility of E.coli and S.aureus to the essential oil of Melaleuca alternifolia (tea-tree oil). Microbios 1995;82:181-5.

[17] Mann CM, Markham JL. A new method for determining the minimum inhibitory concentration of essential oils. J Appl Microbiol 1998;84: $538-544$. 